\title{
Consciência Morfológica, Aspectos Cognitivos da Linguagem e Reconhecimento de Palavras
}

\author{
Márcia Maria Peruzzi Elia da Mota* ${ }^{*, b}$

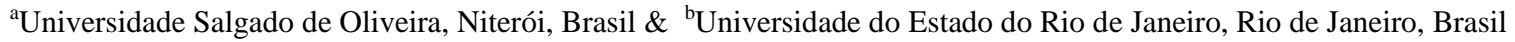

\begin{abstract}
RESUMO
Estudos realizados em línguas alfabéticas mostram que a consciência morfológica está associada ao sucesso no reconhecimento e compreensão de palavras na leitura. Neste estudo, investigamos se o processamento da morfologia contribui para leitura de palavras e se essa contribuição é independente de outros aspectos cognitivos da linguagem, como vocabulário e memória auditiva no português. A amostra é constituída de 52 crianças, sendo 25 do $2^{\circ}$ ano e 27 do $3^{\circ}$ ano, do ensino fundamental. Os resultados das correlações de Pearson mostram que a consciência morfológica está associada à leitura, mas, quando se controla a memória e o vocabulário, esta relação passa a ser significativa só para o terceiro ano.
\end{abstract}

Palavras-chave: consciência metalinguística; consciência morfológica; cognição; alfabetização; leitura.

\section{ABSTRACT \\ Morphological Awareness, Cognitive Aspects of Language and Word Recognition}

Studies carried out in alphabetic languages show that morphological awareness is related to the success in recognizing words and comprehension in reading and spelling. This study investigated whether morphological awareness contributes to reading in Portuguese and whether this contribution is independent of the cognitive aspects of language such as vocabulary and memory. The sample was of 52 children, of those 25 were from the $2^{\text {nd }}$ grade and 27 from $3^{\text {rd }}$ grade. The results of Pearson correlations showed that morphological awareness is associated to reading, but when memory and vocabulary are controlled this relationship is significant for $3^{\text {rd }}$ grade only.

Keywords: metalinguistic awareness; morphological awareness; cognition; literacy; reading.

Morfemas são as menores unidades linguísticas com significado próprio. A habilidade de refletir sobre essas unidades de significado é chamada consciência morfológica e pode ajudar o aprendiz a ler e a escrever (Carlisle, 1995). Isto ocorre porque a ortografia de muitas palavras depende da sua origem. Palavras como "açucarado", que tem ortografia ambígua, podem ser escritas de forma correta, se soubermos sua origem "açúcar". Os significados delas podem ser inferidos, na leitura, se nós soubermos o significado da palavra que as originou. Isso nos leva a crer que a habilidade de refletir sobre os morfemas, também chamada de consciência morfológica, ajudaria na leitura e escrita de palavras, morfologicamente, complexas. Porém, é possível que o processamento morfológico ajude no processamento da leitura, em geral.

Em uma série de estudos que visavam explorar a relação entre a consciência morfológica e a alfabetização em crianças falantes do inglês, Joanne Carlisle mostrou que a habilidade de refletir sobre os morfemas das palavras estava associada ao desempenho na leitura de palavras isoladas, reconhecimento de palavras, à compreensão de leitura (Carlisle, 1995, 2000; Carlisle \& Fleming, 2003) e também ao desempenho

\footnotetext{
Endereço para correspondência: Márcia Maria Peruzzi Elia da Mota, Universidade Salgado de Oliveira, Rua Marechal Deodoro, 217, $2^{\circ}$ andar, Centro, Niterói, RJ, CEP 24030-060. E-mail: mmotapsi@ gmail.com.
} 
da escrita (Carlisle, 1988, 1996). Além dos estudos de Carlisle, outras pesquisas realizadas com falantes do inglês obtiveram resultados semelhantes, tanto no âmbito da leitura (Deacon \& Kirby, 2004; Nagy, Berninger, \& Abbot, 2006) como nas investigações sobre a escrita (Deacon \& Bryant, 2005; Nunes, Bindman, \& Bryant, 1997).

O inglês é uma língua com ortografia muito irregular, com muitas palavras irregulares. Palavras irregulares são palavras que não obedecem a regras de correspondência entre letra e som. Muitas irregularidades no inglês podem ser atribuídas à manutenção da estrutura morfológica das palavras. Precisamos saber como a consciência morfológica contribui para a leitura em línguas com ortografias mais regulares, como o português.

Mann (2000) argumenta que, quando as palavras podem ser escritas ou lidas por correspondência entre letra e som, o processamento morfológico pode ter um peso menor do que o processamento fonológico, nessas ortografias. Contudo, Lehtonen e Bryant (2005) afirmam que não há razão teórica que impeça a formulação da hipótese de que a consciência morfológica contribui para leitura e escrita em línguas cuja ortografia obedeça às regras de correspondência entre letra e som.

Alguns estudos têm demonstrado que a consciência morfológica contribui para leitura e escrita em diferentes ortografias. Colé, Marec-Breton, Royer e Gombert (2003), Sénéchal, Basque e Leclaire (2006) e Duncan, Casalis e Colé (2009) demonstraram essa relação, no francês. Entretanto, o francês é uma língua com muitas irregularidades ortográficas de base morfológica. O português é uma língua interessante para se investigar a consciência morfológica e sua relação com a leitura e escrita, porquanto tem palavras regulares, em termos de correspondência entre letra e som, e, ao mesmo tempo, uma rica estrutura morfológica.

De fato, vários estudos investigando a relação entre a consciência morfológica e a ortografia de palavras morfologicamente complexas, no português, têm sido realizados. Mota (1996) mostrou que, a partir da segunda série, as crianças são capazes de utilizar regras gramaticais, para decidir a grafia de palavras flexionadas. Em outro estudo, Mota e Silva (2007) observaram que as crianças de primeira série, com os melhores escores nos testes de consciência morfológica: analogia gramatical (adaptado de Nunes e cols., 1997) e morfologia produtiva, (adaptado de Berko, 1958) eram, também, as crianças que escreviam melhor pseudopalavras, com ortografia ambígua (ex.: "muge" -"mugidor").

Estudando a relação entre ortografia e morfossintaxe, Rego e Buarque (1997) mostraram que, no seu estudo, a consciência morfossintática contribuiu para o desempenho das crianças na escrita de palavras morfologicamente complexas. Em outro estudo que investigou a relação entre consciência morfossintática e desempenho ortográfico de crianças de $2^{\mathrm{a}}$ e $4^{\mathrm{a}}$ séries do ensino fundamental, Queiroga, Lins e Pereira (2006) avaliaram a escrita das crianças, através de ditados de palavras e pseudopalavras, e, para avaliar a consciência morfossintática, utilizaram uma tarefa de analogia gramatical. Os resultados deste estudo mostraram uma evolução entre as séries, na escrita de palavras e pseudopalavras e na explicitação do conhecimento morfossintático. Encontrou-se, também, um efeito facilitador do conhecimento morfossintático, para a escrita.

Uma fraqueza dos estudos feitos em ortografias regulares reside no fato de que estudam a relação entre a consciência morfológica e a escrita de palavras cujas grafias dependem de regras morfológicas (ex.: "laranja" - "laranjeira"). Também, poucos estudos focaram no caso específico da leitura. Uma exceção foi o estudo feito por Mota e cols. (2009), que mostrou uma relação entre a consciência morfológica e a leitura contextual. As autoras investigaram a relação entre a leitura contextual e consciência morfológica, numa amostra constituída por 42 crianças de segundo e terceiro anos. Mesmo controlando a memória auditiva, os escores da tarefa de analogia gramatical continuaram se correlacionando, de forma positiva e significativa, com uma das tarefas de leitura contextual. No entanto, este resultado só ocorreu com as crianças do terceiro ano e podem apontar um padrão de desenvolvimento. No início da alfabetização, as crianças não utilizam as habilidades morfológicas para a leitura, mas, à medida que progridem na escolarização, essa habilidade pode passar a ser importante.

Os resultados de Mota e cols. (2009) nos dão alguma informação sobre esta relação entre a leitura e a consciência morfológica. Porém, ainda precisamos conhecer mais sobre essa relação. A questão que levantamos é se é possível que a consciência morfológica contribua para a capacidade das crianças de reconhecerem palavras. 
A consciência morfológica é parte de uma habilidade mais geral, chamada habilidade metalinguística. As habilidades metalinguísticas estão associadas ao desenvolvimento linguístico. Nunes e Bryant (2006) mostram que o desenvolvimento do vocabulário das crianças está associado ao desenvolvimento da consciência morfológica. Além do vocabulário, a memória de dígitos é uma medida de processamento fonológico e está associada ao desempenho em várias tarefas cognitivas (Eysenk \& Keane, 2008). Nesse estudo, investigamos a relação entre consciência morfológica e leitura, através de correlações. Porém, correlações não controlam o efeito de variáveis estranhas. Parte da associação entre a consciência morfológica e a leitura pode ser influenciada pela variância partilhada pelo vocabulário e memória. Correlações parciais foram realizadas, para controlar esses fatores.

\section{MÉTODO}

\section{Participantes}

A amostra é constituída de 52 crianças, sendo 25 do $2^{\circ}$ ano e 27 do $3^{\circ}$ ano, ambos do ensino fundamental de uma escola pública federal, situada na região urbana de Juiz de Fora. A média da idade das crianças do $2^{\circ}$ ano foi de 95,92 meses (dp 3.85) e a do $3^{\circ}$ ano, 106,59 meses (dp. 3.94). Foi coletado o Termo de Consentimento Livre e Esclarecido, que deveria ser assinado pelo responsável, do qual dependeu a participação da criança no estudo.

\section{Instrumentos}

\section{A) Tarefa de Analogia Gramatical (adaptada de Nunes e cols., 1997)}

Essa tarefa foi utilizada para analisar o grau de desenvolvimento do conhecimento da consciência morfológica das crianças participantes. Adaptamos a tarefa inicial de Nunes, Bindman e Bryant (1997), para focar mais especificamente na morfologia derivacional do português. Nesta tarefa, a criança tinha que produzir uma palavra morfologicamente complexa, a partir de uma palavra alvo, aplicando a mesma relação de derivação de um par previamente dado, como, por exemplo, "pedra - pedreiro, leite - ?" e, assim, sucessivamente, com os outros pares. Dez itens foram criados.

\section{B) Tarefas de Decisão Morfológica - raiz}

Essa tarefa foi outra utilizada para analisar o grau de desenvolvimento do conhecimento da consciência morfológica das crianças participantes. Nesta tarefa, a criança tinha que decidir qual palavra era da mesma família que a palavra alvo. Os pares de palavras foram pareados pelo número de letras e pela frequiência de ocorrência na escrita (Pinheiro, 1996; tabela para primeira série). Como não há índices de familiaridade para o português, as palavras foram pareadas por frequência, para garantir, minimamente, um equilíbrio na familiaridade das palavras. A lista de palavras consistia de dez grupos de três palavras envolvendo prefixos e dez grupos de palavras envolvendo sufixos.

\section{C) Teste de Leitura- Teste de Desempenho Escolar - TDE (Stein, 1994)}

Os itens de leitura de palavras isoladas do Teste de Desempenho Escolar foram aplicados para avaliar o desempenho na leitura e escrita, das crianças. Por meio deste instrumento, que apresenta propriedades psicométricas satisfatórias, incluindo evidências de validade de critério e bom índice de consistência interna $(\alpha>0,70)$, foi avaliado o desempenho na leitura das crianças.

\section{D) Escala de Inteligência Wechsler para crianças - WISC III (Weschler, 1991)}

Os subtestes de Vocabulário, Compreensão e Dígitos foram aplicados. Os escores ponderados foram utilizados. Este teste foi aplicado para controlarmos a influência do desenvolvimento cognitivo na aquisição da leitura e escrita. Os coeficientes de fidedignidade, para esses subtestes, foram calculados pelo método de Guttmam e apresentam bons índices de consistência interna (coeficientes maiores que 0,60 ).

\section{Procedimentos}

As crianças foram avaliadas individualmente, em duas sessões de 30 a 40 minutos. Na primeira, foram realizados os testes de analogia gramatical e dois subtestes do WISC (Vocabulário e Dígitos). Na segunda sessão, foram aplicados o Ditado de Palavras, as tarefas de decisão morfológica e dois subtestes do Teste de Desempenho Escolar. Este estudo concentra apenas a análise dos resultados no Ditado de Palavras.

Nas Tarefas de Decisão Morfológica - raiz, era dada a seguinte instrução: "algumas palavrinhas são da mesma família de outras. Por exemplo, a palavra 'conta' e a palavra 'reconta' são da mesma família. Já a palavra 'bola' e 'rebola' não são da mesma família. Eu vou falar, para você, uma palavra e depois vou falar mais outras duas e você vai me dizer qual das 
duas é da mesma família da primeira”. Por fim, realizava-se um exemplo com a criança: "a palavra 'gela' é da mesma família que 'congela' ou 'conversa'?" Caso a criança errasse, explicava-se a forma correta e, se acertasse, iniciava-se a tarefa. Depois do exemplo, iniciava-se a aplicação da tarefa, mesmo que a criança não conseguisse acertá-lo.

$\mathrm{Na}$ Tarefa de Analogia Gramatical (adaptada de Nunes e cols., 1997), foi informado às crianças que muitas palavras poderiam ser relacionadas. A aplicadora procedeu apresentando um par de palavras relacionadas, depois, ela pedia à criança para ouvir uma palavra e que ela criasse outra palavra relacionada com a que era dada, seguindo o modelo do exemplo. A tarefa era iniciada sempre pelo exemplo "pedra pedreiro; leite - ?" e, assim, sucessivamente, eram pronunciadas as demais palavras-alvo.

Os dois subtestes do WISC III (Vocabulário e Dígitos) foram aplicados segundo o procedimento padrão de aplicação. Os dois subtestes do Teste de De- sempenho Escolar também foram aplicados seguindo o procedimento de aplicação do manual.

\section{RESULTADOS}

Em primeiro lugar, exploramos a relação entre as medidas de habilidade cognitiva (memória auditiva e vocabulário), leitura e consciência morfológica, através de correlações simples. Os resultados das correlações de Pearson, realizadas para explorar a relação entre a leitura e o processamento morfológico, mostram correlações fracas, significativas e positivas, entre a leitura e a consciência morfológica ( $\mathrm{r}=0,37$ e $p$ $<0,01)$ e correlações fortes, positivas e significativas, com a tarefa de memória auditiva ( $\mathrm{r}=0,65$ e $p<0,01)$. A correlação entre a tarefa de analogia e de vocabulário foi fraca, mas significativa $(\mathrm{r}=0,33$ e $p<0,01)$. As duas outras tarefas de consciência morfológica não produziram correlações com resultados significativos, com nenhuma das medidas cognitivas ou de leitura. A Tabela 1 mostra o resultado dessas correlações.

Tabela 1

Coeficientes de Correlação de Pearson Para as Tarefas de Consciência Morfológica, Vocabulário, Dígitos e Leitura de Palavras

\begin{tabular}{|c|c|c|c|c|c|}
\hline & $\begin{array}{l}\text { Decisão } \\
\text { Prefixo }\end{array}$ & $\begin{array}{l}\text { Decisão } \\
\text { Sufixo }\end{array}$ & Vocabulário & Dígito & TDE leitura \\
\hline Analogia &,- 10 & 0,01 & $0,33^{*}$ & $0,35^{* *}$ & $0,37^{* *}$ \\
\hline $\begin{array}{l}\text { Decisão } \\
\text { Prefixos }\end{array}$ & & $0,48^{* *}$ & $-0,01$ & 0,01 & $-0,11$ \\
\hline $\begin{array}{l}\text { PrefIXos } \\
\text { Decisão }\end{array}$ & & &,- 035 & 0,10 & 0,04 \\
\hline $\begin{array}{l}\text { Sufixo } \\
\text { Vocabulário }\end{array}$ & & & & 0,18 & 0,25 \\
\hline Dígito & & & & & $0,65^{* *}$ \\
\hline
\end{tabular}

$\mathrm{Na}$ segunda parte das análises, exploramos a relação entre a consciência morfológica e a leitura, controlando para as variáveis cognitivas. Antes de fazer essas análises, verificamos que houve diferenças significativas entre os anos escolares, no desempenho na leitura $(\mathrm{t}(50)=-2,8, p=0,007)$. Não seria apropriado entrar com a variável "ano", na análise, porquanto é uma variável dicotômica. Correlações parciais foram realizadas para cada ano, isoladamente.

Uma correlação parcial entre a medida de analogia e a leitura foi realizada, controlando-se, para a variância compartilhada, com a memória e dígitos, para cada ano. $\mathrm{O}$ resultado, para o primeiro ano, mostra que a consciência morfológica não apresenta correlações significativas, quando dígito e memória são controlados $(\mathrm{r}=0,09$ e $p=0,7)$. Para o segundo ano, o resultado mostra que a associação entre a consciência morfológica e a leitura é significativa, mesmo que se controle a memória e vocabulário $(\mathrm{r}=0,45$ e $p=0,02)$.

\section{DISCUSSÃO}

O presente estudo se propôs a investigar a relação entre a consciência morfológica, aspectos cognitivos da linguagem e a leitura de palavras. Os resultados mostram que o processamento fonológico, medido pela tarefa de dígito, contribui para a leitura. Esses 
resultados são congruentes com os de vários estudos que demonstram que essa habilidade tem um papel fundamental na aquisição da língua escrita, isto é, na aprendizagem da leitura e escrita (Bradley \& Bryant, 1983; Capovilla \& Capovilla, 2000; Cardoso-Martins, 1995; Cuninghan, 1990; Guimarães, 2003; Maluf \& Barrera, 1997; Santos, 1996). É interessante ressaltar que essa relação se manteve mesmo depois de controlarmos o desempenho nas tarefas de vocabulário e na consciência morfológica, demonstrando a força dessa habilidade para aquisição da leitura.

A relação entre a consciência morfológica e a leitura foi demonstrada através dos índices de correlação obtidos. As correlações positivas e significativas entre a medida de leitura e as medidas de analogia gramatical foram fracas, mas significativas. As crianças que tiveram maiores escores no Teste de Desempenho Escolar foram aquelas com maiores escores na tarefa de consciência morfológica. Estes resultados sugerem que, além do processamento fonológico, o processamento morfológico contribui para leitura. São compatíveis, também, com resultados realizados, investigando a relação entre a leitura contextual e a consciência morfológica (Mota e cols. 2009). Embora correlações não estabeleçam relações de causa e efeito, estes resultados indicaram que essa questão deveria ser mais bem explorada. A consciência morfológica é, usualmente, associada à leitura contextual, pois as regras sintáticas e semânticas de um texto podem ajudar a criança a prever as palavras que não conhece, num texto. É interesse que a consciência morfológica tenha contribuído para o reconhecimento de palavras. Uma hipótese é a de que o processamento morfológico entraria em ação, quando as crianças precisam utilizar seu conhecimento sobre a formação de palavras, quando precisam ler palavras que não conhecem.

Correlações parciais mostraram que a relação entre o processamento morfológico e a leitura se mantém, mesmo controlando as variáveis, como a memória auditiva e vocabulário. Os escores da tarefa de analogia gramatical continuam se correlacionando de forma positiva e significativa com a leitura, mesmo quando os controles citados foram efetuados. Esses resultados indicam uma relação específica entre a consciência morfológica e a leitura, que independe do processamento fonológico. No entanto, este resultado só ocorreu para as crianças de terceiro ano, o que leva a cogitar se há um padrão no desenvolvimento, como o proposto por Nunes e cols. (1997), para escrita na leitura.
Esses autores propuseram que, no início da aquisição da escrita, a consciência morfológica teria um peso menor do que a consciência fonológica, porque as crianças estão aprendendo as regras de correspondência entre letra e som. À medida que as crianças avançam e têm que aprender regras ortográficas mais complexas, a consciência morfológica passa a ter um peso maior na aquisição da escrita. Correlações não estabelecem medidas de causa e efeito. Estudos futuros devem ser realizados para que se possa estabelecer se há uma relação causal entre a consciência morfológica e a leitura e se há diferenças de peso, na contribuição dessas habilidades metalinguísticas.

Um último ponto que precisa ser discutido diz respeito às características linguísticas do português. Uma das questões levantadas, nessa área, é se, no português, língua com ortografia mais transparente do que a do inglês e francês, a consciência morfológica contribuiria para aquisição da língua escrita. Nossos dados apontam que sim. Sugerem, também, que as etapas de desenvolvimento da língua escrita são semelhantes, partindo-se do processamento alfabético da língua escrita para uma análise mais complexa da ortografia do português do Brasil.

\section{REFERÊNCIAS}

Berko, J. (1958). The child learning of English morphology. Word, 14, 150-177.

Bradley, L., \& Bryant, P. (1983). Categorizing sounds and learning to read: A causal connection. Nature, 301, 419-421.

Capovilla, A., \& Capovilla, F. (2000). Efeitos do treino de consciência fonológica em crianças com baixo nível sócio-econômico. Psicologia: Reflexão e Crítica, 13, 7-24.

Cardoso-Martins, C. (1995). Consciência fonológica e alfabetização. Petrópolis: Vozes.

Carlisle, J. (1988). Knowledge of derivational morphology and spelling ability in fourth, six, and eight graders. Applied Psycholinguistics, 9, 247-266.

Carlisle, J. (1995). Morphological awareness and early reading achievement. Em L. Feldman (Org.), Morphological aspects of language processing (pp. 189-211). Hillsdale: Lawrence Erlbaum.

Carlisle, J. (1996). An exploratory study of morphological errors in children`s written stories. Reading and Writing: An Interdisciplinary Journal, 8, 61-72.

Carlisle, J. (2000). Awareness of the structure and meaning of morphologically complex words: Impact on reading. Reading and Writing: An Interdisciplinary Journal, 12, 169-190.

Carlisle, J., \& Fleming, J. (2003). Lexical processing of morphologically complex words in the elementary years. Scientific Studies of Reading, 7, 239-253. 
Colé, P., Marec-Breton, N., Royer, C., \& Gombert, J. E. (2003). Morphologie des mots et apprentissage de la lecture. Reeducation Orthophonic, 213, 57-60.

Cunningham, A. E. (1990). Implicit versus explicit instruction in phonemic awareness. Journal of Experimental Child Psychology, 50, 426-444.

Deacon, S., \& Bryant, P. (2005). What young children do and do not know about the spelling of inflections and derivations. Developmental Science, 8, 583-594.

Deacon, S., \& Kirby, J. (2004). Morphological awareness: Just "more phonological"? The roles of morphological and phonological awareness in reading development. Applied Psycholinguistics, 25, 223-238.

Duncan, L., Casalis, S., \& Colé, P. (2009). Early metalinguistic awareness of derivational morphology: Observations from comparison of English and French. Applied Psycholinguistics, $30,405-440$.

Eysenck, M., \& Keane, M. (2008). Psicologia cognitiva: Um manual introdutório (W. Gesser \& M. H. F. Gesser, Trad.). Porto Alegre: Artmed.

Guimarães, S. (2003). Dificuldades no desenvolvimento da lectoescrita: O papel das habilidades metalinguísticas. Psicologia: Teoria e Pesquisa, 19, 33-45.

Lehtonen, A., \& Bryant, P. (2005). Active players or just passive bystanders? The role of morphemes in spelling development in a transparent orthography. Applied Psycholinguistics, 26, 137155.

Maluf, M. R., \& Barrera, S. D. (1997). Consciência fonológica e linguagem escrita em pré-escolares. Psicologia: Reflexão $e$ Crítica, 10, 125-145.

Mann, V. (2000). Introduction to special issue on morphology and the acquisition of alphabetic writing systems. Reading and Writing: An Interdisciplinary Journal, 12, 143-147.

Mota, M. (1996). Children's role of grammatical rules in spelling. Tese de doutorado não-publicada, Universidade de Oxford.

Mota, M., Lisboa, R., Dias, J., Gontijo, R., Paiva, N., MansurLisboa, S., Silva, D., \& Santos, A. (2009). Relação entre consciência morfológica e leitura contextual medida pelo teste de Cloze. Psicologia: Reflexão e Crítica, 22, 223-229.
Mota, M., \& Silva, K. (2007). Consciência morfológica e desenvolvimento ortográfico: Um estudo exploratório. Psicologia em Pesquisa, 1, 86-92.

Nagy, W., Berninger, V., \& Abbot, R. (2006). Contributions of morphology beyond phonology to literacy outcome of upper elementary and middle-school students. Journal of Educational Psychology, 98, 134-147.

Nunes, T., Bindman, M., \& Bryant, P. (1997). Morphological strategies: Developmental stages and processes. Developmental Psychology, 33, 637-649.

Nunes, T., \& Bryant, P. (2006). Improving literacy by teaching morphemes. London: Routledge.

Pinheiro, A. M. V. (1996). Contagem de freqüência de ocorrência de palavras expostas a crianças na faixa pré-escolar e séries iniciais do $1^{\circ}$ grau [Software]. São Paulo: Associação Brasileira de Dislexia.

Queiroga, B., Lins, M., \& Pereira, M. (2006). Conhecimento morfossintático e ortografia em crianças do ensino fundamental. Psicologia: Teoria e Pesquisa, 22, 95-99.

Rego, L., \& Buarque, L. (1997). Consciência sintática, consciência fonológica e aquisição de regras ortográficas. Psicologia: Reflexão e Crítica, 10, 199-217.

Santos, A. A. A. (1996). A influência da consciência fonológica na aquisição da leitura e da escrita. Em F. F. Sisto, G. C. Oliveira, L. D. T. Fini, M. T. C. C. Souza, \& R. P. Brenelli (Orgs.), Atuação psicopedagógica e aprendizagem escolar (pp. 213-247). Petrópolis: Vozes.

Sénéchal, M., Basque, M., \& Leclaire, T. (2006). Morphological knowledge as revealed in children's spelling accuracy and reports of spelling strategies. Journal of Experimental Child Psychology, 95, 231-234.

Stein, L. M. (1994). TDE - Teste de desempenho escolar: Manual para aplicação e interpretação. São Paulo: Casa do Psicólogo.

Wechsler, D. (1991). WISC-III: Escala de inteligência Weschsler para crianças. São Paulo: Casa do Psicólogo.

Recebido: 04/10/2009

Última revisão: 01/09/2010

Aceite final: $23 / 10 / 2010$ 